\title{
INMIGRACIÓN EN TIEMPOS DE CRISIS: DINÁMICAS DE MOVILIDAD EMERGENTES Y NUEVOS IMPACTOS SOCIALES
}

\author{
Ana LóPEZ-SALA* y LaURa Oso**
}

\section{LA CRISIS ECONÓMICA COMO MOTOR DE CAMBIO EN LOS ESTUDIOS MIGRATORIOS EN ESPAÑA: NUEVOS ESCENARIOS ANALÍTICOS DE LA MOVILIDAD EN TIEMPOS DE CRISIS}

La crisis económica experimentada por España desde 2009 ha inducido un cambio de ciclo migratorio (Domingo y Recaño, 2010; Reher, Requena y Sanz, 2011). Tras algo más de dos décadas en las que España se consolidó como uno de los principales Estados receptores del mundo, los registros estadísticos de principios de la década han puesto de relieve el impacto de la crisis en el volumen y la direccionalidad de los flujos subrayando la interacción entre movilidad y dinámica económica. Este cambio ha combinado tres tipos de pautas. En primer lugar una ralentización de las nuevas llegadas; en segundo lugar, un incremento de las salidas de ciudadanos españoles, especialmente desde 2011 y; en tercer lugar, una demora del retorno de los españoles residentes en el exterior (Domingo y Sabater, 2013; Domingo, Sabater y Ortega, 2014; Domínguez-Mújica, Guerra y Parreño, 2014; Sánchez-Montijano y Alonso, 2014). A ello se añaden los indicios, amplificados por los medios de

* Consejo Superior de Investigaciones Científicas (CSIC).

** ESOMI-Universidade da Coruña (UDC). 
comunicación, sobre los procesos de reemigración y retorno entre los residentes extranjeros (Parella y Petrof, 2014; Boccagni y Lagomarsino, 2011). En definitiva, nos encontramos en la actualidad ante un panorama analítico mucho más complejo, que transciende el enfoque unidireccional centrado en exclusiva en la conformación de España como destino migratorio y donde ha cobrado protagonismo el uso de una perspectiva más holística en el estudio de las nuevas constelaciones de la movilidad y sus impactos sociales.

Este cambio de ciclo migratorio ha suscitado una readaptación al objeto de estudio, por parte de los estudiosos de esta realidad. Frente a la época de la bonanza, en la que el centro de atención eran "las llegadas", la crisis ha sido, sin duda, la gran protagonista en la producción científica sobre las migraciones en los últimos años; emergiendo como un nuevo escenario de indagación académica en el VII Congreso sobre las Migraciones Internacionales en España (Blanco, 2014). Y siendo igualmente objeto de sesiones específicas en distintos grupos de trabajo de los congresos nacionales de Sociología y de Antropología. Su relevancia se ha reflejado también en los Anuarios sobre la Inmigración que edita desde hace casi una década la Fundación CIDOB (Aja, Arango y Oliver, 2009, 2010 y 2012); en la inclusión, desde finales de la década pasada, de secciones monográficas en el Anuario de Relaciones Laborales en España (Ferrer Sais, Santos Ruesga y Resa Nestares, 2010, 2011); en diversas compilaciones específicas realizadas en publicaciones económicas (Lázaro Alquézar, Sánchez y Simón, 2012; De Arce, 2010; Matía Portilla y Álvarez Rodríguez, 2012); en ediciones de carácter interdisciplinar (Torres y Gadea, 2015; Durán Ruiz, Fernández-Avilés, Guillén, Moreno y Ortega, 2011), así como en publicaciones que se han centrado en análisis comparativos sobre determinadas comunidades en distintos entornos geográficos (Aysa-Lastra y Cachón, 2015; Sassone y Yépez, 2014).

Este monográfico pretende contribuir, desde una perspectiva plural, a este profuso panorama de la investigación nacional sobre las migraciones en un contexto de crisis que, sin duda, tiene por delante enormes retos. Esta tarea no habría sido posible sin la excelente acogida que la Revista Migraciones mostró ante nuestra iniciativa, así como sin el interés, el trabajo y la dedicación de los autores que han participado con sus contribuciones. El monográfico incluye tres áreas principales de análisis. La primera se detiene en los efectos de la crisis sobre las dinámicas laborales de los migrantes. La segunda, 
subraya y explora sus estrategias adaptativas, que combinan tanto iniciativas como motivaciones. El monográfico termina por abordar, los impactos sociales de la crisis en distintas esferas de la realidad social, aludiendo a la reconfiguración de la convivencia en este nuevo ciclo.

\section{MIGRACIONES Y CRISIS ECONÓMICA: ALGUNAS CERTEZAS Y MÚLTIPLES INCERTIDUMBRES}

\subsection{Vulnerabilidad y resiliencia de los migrantes ante un mercado de trabajo segmentado y precarizado}

El impacto de la crisis en el empleo de la población inmigrante ha suscitado especial interés por parte de los investigadores, que han abordado este objeto a través de la explotación de fuentes secundarias, y sobre todo de la Encuesta de Población Activa (Muñoz Comet, 2012 y 2013; Garrido, Miyar y Muñoz Comet, 2010; Rodríguez-Planas y Nollenberger, 2014). Destacan, entre las principales aportaciones al respecto, los trabajos de Oliver i Alonso (2011, 2013 y 2014), que ha llevado a cabo un seguimiento de la evolución del mercado laboral de la inmigración desde el inicio de la crisis financiera en 2007, constatando cómo la pérdida de empleo inmigrante alcanzó su máximo en lo que este autor ha llamado la «segunda recesión», entre junio de 2011 y marzo de 2013, periodo en el cual casi 500.000 migrantes perdieron sus empleos. Este autor muestra también un cambio en la dinámica del trabajo desde marzo de 2013 que apunta al final de la dura recesión, con una relativa mejora, debido sobre todo al aumento del empleo de inmigrantes autónomos y la reducción de la intensa caída del trabajo asalariado temporal (Oliver i Alonso, 2014). En cuanto a los vínculos entre educación y empleo algunos autores han constatado además cómo la educación protege menos a los migrantes que a los nativos del desempleo, en especial a los procedentes de África y América Latina (Cebolla-Boado, Miyar y Muñoz Comet, 2015).

Uno de los debates que ha suscitado la producción científica es el que intenta identificar si la población inmigrante en España ha sido más vulnerable o más resiliente ante la crisis global. Esta es una problemática que han abordado, por ejemplo, en un libro de 
reciente edición, Aysa-Lastra y Cachón, comparando los casos de España y EEUU (Aysa-Lastra y Cachón, 2015). En esta línea de análisis se enmarca el artículo de Fernando Gil-Alonso y Elena VidalCoso para este monográfico. Como nos recuerdan estos autores el debate científico suscitado discurre, por un lado, entre los investigadores que argumentan que en un contexto de crisis los inmigrantes son el colectivo más vulnerable, al ser los primeros expulsados del mercado de trabajo (buffer theory), regresando a su país y liberando puestos de trabajo para los autóctonos. Y, por otro, aquellos especialistas que consideran que los trabajadores autóctonos son los más perjudicados debido a que en un contexto de mercados de trabajo muy segmentados, los migrantes son más capaces de ajustarse a la precarización en momentos de recesión (Fielding, 2010). Una de las aportaciones más originales del texto de Gil-Alonso y Vidal-Coso resulta de llevar a cabo una periodización de la crisis, sacando a la luz cómo la mayor o menor vulnerabilidad/resiliencia de los inmigrantes extranjeros, en comparación con los trabajadores autóctonos, ha ido variando según las distintas etapas de la recesión; articulando, así, estas dos perspectivas teóricas, que para el caso español, no resultan ser excluyentes. Si en la primera mitad de la crisis, los extranjeros fueron más resilientes, en la segunda parte, desde mediados de 2011, y sobre todo desde principios de 2013, han pasado a ser más vulnerables.

El enfoque de género también destaca en la aportación de GilAlonso y Vidal-Coso, en la línea de otros trabajos previos que han sacando a la luz cómo la crisis ha impactado más en el empleo masculino inmigrante (Muñoz Comet, 2013) o que ponen de manifiesto, comparando el caso de España y Portugal, cómo las sucesivas crisis que se han desarrollado en el sector de la construcción en estos dos países, desde los años noventa hasta la actualidad, vienen acompañadas de una feminización del stock de inmigrantes, durante los periodos de recesión (Oso y Catarino, 2014).

En esta línea de análisis, Gil Alonso y Vidal-Coso muestran que en la primera etapa, hasta el verano de 2011, la crisis afectó fundamentalmente a los hombres, al concentrarse la pérdida de empleo en el sector de la construcción tras el pinchazo de la burbuja inmobiliaria. No obstante, a partir del tercer trimestre de 2011, el impacto de la crisis llega a otros sectores de actividad (administración pública, educación, sanidad), sufriendo las mujeres una pérdida importante de puestos de trabajo. Esto lleva a los autores a retomar, para esta 
última fase de la crisis, la buffer theory, según la cual, los migrantes saldrían de algunos sectores laborales y puestos de trabajo que están volviendo a ser ocupados por los autóctonos, tales como los servicios personales, el servicio doméstico y la agricultura.

El artículo de María Elena Gadea, Carlos de Castro, Andrés Pedreño y Natalia Moraes para este monográfico, ahonda precisamente en este último sector, en concreto en la agricultura intensiva de exportación, que ha basado su crecimiento en actividades intensivas en mano de obra y en donde ha jugado un papel estratégico la inserción socio-laboral de trabajadores inmigrantes. Aunque ya se observaba una sobrerrepresentación de los trabajadores extranjeros en la agricultura española antes de la crisis, esta sobrerrepresentación ha crecido durante los años de recesión, alcanzando en 2013 el $22,5 \%$ del total de afiliados. En los primeros años de la recesión se ha producido una vuelta a la agricultura de trabajadores extranjeros que habían perdido su empleo en la construcción, la industria y los servicios.

El artículo se detiene en el caso de la región de Murcia, una zona emblemática de agricultura intensiva de exportación. Basándose en el trabajo de campo realizado entre 2012 y 2014, los autores muestran que durante los años de crisis económica se ha producido también un retorno significativo de trabajadores españoles a la agricultura murciana, con una presencia similar a la observada hace una década, algo que ha producido un cambio en el principio de complementariedad que había regido la posición de inmigrantes y autóctonos en el mercado de trabajo agrícola. Este cambio también ha sido inducido por transformaciones en la política española (López-Sala, 2013), en especial a través de la contención de la contratación en origen de trabajadores extranjeros para el sector agrícola español (Gualda, 2012; López-Sala y Sánchez-Montijano, 2014). En cuanto al empleo de los extranjeros en la agricultura de la región de Murcia, la investigación llevada a cabo por los autores constata que su inserción laboral se ha caracterizado por altos niveles de temporalidad y de rotación, así como por la importancia de la contratación a través de empresas de trabajo temporal, procesos que en el contexto de crisis se han intensificado. La crisis ha producido también una degradación de las condiciones laborales de los trabajadores, en especial, una proliferación de las irregularidades laborales, la intensificación de los ritmos de trabajo y la generalización de la precariedad laboral. 
El debate en torno a la segmentación del mercado de trabajo español sale también a la luz en el artículo de Concepción Carrasco Carpio y Carlos García Serrano. Estos autores abordan cómo la estructura ocupacional segmentada hacia puestos de naturaleza manual para los extranjeros nacidos fuera de España ha potenciado una pérdida de ocupación en la biografía laboral de las cohortes de individuos más jóvenes en el periodo recesivo. La originalidad de esta aportación consiste en utilizar, a través de la explotación de los microdatos de la EPA, la metodología de cohortes ficticias, que permite estudiar las trayectorias de empleo de los migrantes a lo largo de su biografía laboral. Su artículo se enmarca, por tanto, en los trabajos que buscan dar cuenta del impacto de la crisis en el empleo inmigrante, a partir de un enfoque longitudinal de las trayectorias laborales (Miguelez Lobo, López-Roldán y Alós-Moner Vila, 2014).

Concepción Carrasco Carpio y Carlos García Serrano buscan profundizar en cómo la segmentación laboral influye en las trayectorias de empleo de los individuos a lo largo del tiempo. Y concluyen que el cambio de las condiciones económicas a partir del año 2008, que perduran hasta 2013, ha golpeado especialmente a la población extranjera, lo que ha dado lugar a una pérdida de la ocupación en la biografía de los individuos (en términos de reducción de sus tasas de ocupación respecto a las que tuvieron personas de cohortes anteriores con su misma edad), siendo, por tanto, el impacto de la crisis más fuerte para las cohortes de edad más jóvenes. Pero siempre hay una excepción y la comunidad china vuelve a serlo. Parece que en este contexto de crisis que está azotando con dureza a los inmigrantes, ellos siguen yendo a contracorriente, como señalan Joaquín Beltrán y Amelia Saíz, en su artículo para este monográfico. Y es que el concentrar su actividad laboral en el marco de nichos económicos étnicos, parece haber "protegido" a los trabajadores chinos en España, siendo un ejemplo, en comparación con otros colectivos, de resilencia ante la crisis. En efecto, los chinos siguen creciendo en sus efectivos, pero también en sus actividades empresariales. Pero ¿cuál ha sido el secreto de los miembros de la comunidad china? Beltrán y Saíz argumentan que esta comunidad ha mantenido, consolidado y promovido una dinámica que ya había desarrollado anteriormente a la crisis, y que consiste en tres estrategias: 1) la puesta en marcha de empresas familiares; 2) la búsqueda, inserción y explotación de nuevos nichos económicos; y 3) la intensificación del transnacionalismo en la actividad empresarial. La diversificación de actividades 
productivas en el seno familiar, que les protege frente a las dificultades económicas, incluye la movilización, a través del espacio transnacional, de capitales, información y mano de obra. Estas actividades empresariales chinas se entienden en su relación con el ciclo de vida de la familia, incluidas las actividades transnacionales.

\subsection{Estrategias de los migrantes ante la crisis. Una reacción adaptativa y multifacética todavía por explorar}

Como hemos visto en el apartado anterior no es posible entender el impacto de la crisis si no se toma en cuenta la importancia de la familia como unidad de análisis; enfoque sobre el cuál ahonda Jesús Sanz en su artículo para este monográfico. Y es que esta dimensión ha sido, por el momento, menos trabajada en la producción científica española, a excepción de algunos trabajos que han mostrado las estrategias familiares que están poniendo en marcha los hogares migrantes para hacer frente a la recesión. Estas estrategias incluyen, entre otras iniciativas, la incorporación de nuevos miembros de la familia al mercado de trabajo, estrategias de ahorro y de reestructuración del envío de remesas, el retorno permanente o temporal o las remigraciones selectivas por género a terceros países (Juliano, 2012; Pedone, Agrela y Gil-Araujo, 2012: Herrera, 2012; Viruela, 2013; Torres, 2014; Oso y Martínez-Buján, 2014). En esta línea de análisis, Moser y Horn (2013), a partir de datos longitudinales, señalan cómo, a pesar del contexto macroeconómico desfavorable, un pequeño grupo de migrantes de Guayaquil asentados en Barcelona, han puesto en marcha, a través de la formalización de su estatuto legal o de la ciudadanía, un "círculo virtuoso" de consolidación de un portafolio interrelacionado de activos (capital financiero, físico, etc.), que les ha permitido, a pesar de la crisis, permanecer en España.

Jesús Sanz, ahonda en esta aproximación partiendo de un trabajo etnográfico multisituado que incluye entrevistas a los migrantes y sus familias en España y Ecuador. Para ello analiza el proyecto migratorio y el retorno, como parte de un mismo proceso que se construye sobre la base de la articulación de las estrategias productivas y reproductivas del hogar, entendido este como un espacio de negociación. El artículo se detiene también en el impacto que tienen el marco normativo y la situación económica de los países de origen y destino en la configuración de estas estrategias. Su trabajo pone de 
manifiesto cómo los hombres ecuatorianos han sido los que han encabezado el proceso de retorno, así como la reemigración a terceros países, en la línea de los trabajos ya citados. Por otro lado, identifica dos trayectorias migratorias. En primer lugar, el no retorno, protagonizada por los migrantes y sus familiares que no desean volver a Ecuador y que priorizan el asentamiento en España u optan por la emigración a un tercer país. Esta trayectoria está muy determinada por el arraigo y el deseo de permanencia en los hijos. Las estrategias puestas en marcha por estas familias han consistido en múltiples acciones, que abarcan la venta de propiedades en Ecuador o la recepción de remesas desde este país, por ejemplo. Los que optan por emigrar a un tercer país suelen ser personas sin familiares a cargo y se trata de una opción pensada como temporal. En segundo lugar, encontramos la trayectoria del retorno, que suele darse en tres modalidades: retorno selectivo, retorno planificado y retorno sobrevenido. En cualquiera de los casos, estas trayectorias son el resultado de la rearticulación de la esfera productiva y reproductiva en el seno de la unidad familiar, donde cuestiones como el contexto laboral o la organización de los cuidados (educación de los hijos, formación académica y socialización, etc.) son fundamentales a la hora de tomar la decisión de retornar o no.

Y es que la crisis económica ha suscitado un enorme interés por el estudio del retorno de los migrantes, tanto en España (Cavalcanti y Parellá, 2013), como en el plano internacional (Bastia, 2011). En España este interés ha descansado en dos elementos. En primer lugar, en la asunción por parte de la opinión pública, los medios de comunicación y una parte de los representantes políticos, de que la crisis y las dificultades de los migrantes inducirían la alteración de los proyectos migratorios e impulsarían un nutrido regreso al país de origen. En segundo lugar, como se observa a nivel internacional, el contexto de crisis ha creado un escenario propicio para el resurgimiento del retorno como objeto de estudio, que ha experimentado un revival en la teoría migratoria, como ya sucedió durante la década de los setenta (Cerase, 1974). En este contexto se enmarca el trabajo de Diego López de Lera y Antía Pérez Caramés, para este número monográfico. Estos autores abordan en su artículo los procesos relativos a la motivación y la toma de decisiones, repasando la influencia, entre otros, de los enunciados de la teoría del capital social y de la movilización de recursos. El artículo se detiene en el caso de las dos comunidades con mayor presencia en España: la rumana 
y la ecuatoriana. El análisis pormenorizado de las estimaciones del retorno a través de la explotación de los microdatos de la Estadística de Variaciones Residenciales (EVR) indica, según los autores, que pese al previsible impacto de la recesión en este proceso, la magnitud del retorno encaja dentro de las estimaciones realizadas por los organismos internacionales, en ausencia del efecto perturbador de la crisis. Sus principales conclusiones apuntan a la importancia que tiene la organización del trabajo reproductivo y sus crisis, especialmente notoria como motivación del retorno de los migrantes rumanos, coincidiendo, en este aspecto, con los resultados del trabajo de Jesús Sanz. A esto hay que añadir, sostienen los autores, la relativa flexibilidad que aporta la seguridad administrativa, como se evidencia en el caso de la comunidad ecuatoriana. Como vemos, la pluralidad de factores activadores y motivadores del retorno hace necesario, en su opinión, superar viejas dicotomías explicativas en torno al eje éxito-fracaso. Además la crisis económica, y las dificultades laborales, parecen simplemente haber acelerado, no transformado, la toma de decisiones sobre el retorno. El estudio comparado muestra, para concluir, que ni la situación administrativa, ni la posición económica o familiar son buenas predictoras de la decisión de retornar.

\subsection{Convivencia vecinal, actitudes ante la inmigración e inserción escolar: ¿competencia ante los recortes en servicios públicos?}

Las estrategias de movilidad que están poniendo en marcha los inmigrantes ante la crisis también son puestas de relieve por Francisco Torres Pérez, Albert Moncusí Ferré y Fernando Osvaldo Esteban, en su trabajo, para este monográfico, sobre crisis, convivencia multicultural y "efectos de barrio"; y en donde cobran protagonismo algunas de las dimensiones locales de los vínculos entre crisis económica y estrategias residenciales. El trabajo se detiene en dos enclaves de Valencia, Russafa y Els Orriols; el primero, un barrio céntrico popular, en proceso de gentrificación y el segundo, periférico y de tradición obrera. Estos autores constatan, a través de su investigación, que el impacto de la crisis ha sido mayor para los inmigrantes de estos entornos, por su precaria situación socioeconómica, mayor incidencia de paro, así como resultado de lo que los autores 
denominan efectos "desestabilizantes". Esta situación ha ocasionado una reducción de los vecinos de origen inmigrante en Russafa y Els Orriols que se han trasladado a otros distritos, o han puesto en marcha estrategias de movilidad transnacional, similares a las que anunciaba Jesús Sanz en su artículo. Algunas familias ecuatorianas y bolivianas han retornado, pero otros inmigrantes, sobre todo los varones latinoamericanos, han reemigrado a diversos países europeos, mientras que sus familias continúan residiendo en estos enclaves urbanos. En el caso de las familias marroquíes, se dan casos en los que el padre, en paro, permanece en Valencia, mientras que la mujer y los hijos se han trasladado a Marruecos. En ambos casos, se trata de "estrategias de reproducción familiar transnacional que mantienen la relación con el barrio". En cuanto a las relaciones entre vecinos inmigrantes y autóctonos, sigue dándose "una convivencia pacífica, pero distante", constatándose una creciente competencia en lo referente a los servicios sociales. Así, en palabras de estos autores "los CMSS, Cáritas y otros actores constatan el aumento de competencia entre vecinos de distintos orígenes por ayudas sociales y su expresión en términos de una resignificación negativa del inmigrante, lo que aparece como clara fuente de posibles tensiones etnificadas futuras". Esta tensión o competencia entre autóctonos e inmigrantes, por los servicios públicos, es una de las líneas de investigación emergentes sobre el nexo crisis-migración, que han trabajado igualmente otros autores (Alemán y Soriano, 2013; Pedreño, Moraes y Gadea, 2015).

Las actitudes ante la inmigración es otra de las temáticas claves de análisis entre los investigadores que se han interesado por el impacto de la crisis, no sólo en España, sino en otros países receptores. En España esta tarea ha sido abordada, en gran parte, en estudios basados en encuestas, realizados por diversos observatorios nacionales y regionales (Moreno, 2013; Moreno y Fullaondo, 2011). En términos generales las investigaciones sobre los vínculos entre migración y actitudes han estado profundamente influidos por la teoría del conflicto grupal desarrollada en el trabajo pionero de Allport, a mediados de la década de los cincuenta, y posteriormente reformulada en el influyente artículo de Quillian en los noventa (Allport, 1954; Quilliam, 1995). Es el caso del artículo dedicado en este monográfico a "Los Efectos de la Crisis económica en la molduración y evolución de la opinión pública ante la inmigración”, escrito por $\mathrm{M}^{\mathrm{a}}$ Ángeles Cea D'Ancona. Su objetivo es mostrar y explicar los cambios 
de las actitudes ante la inmigración en tiempos de crisis económica, abarcando aspectos económicos y culturales. Utilizando como marco de referencia la teoría del conflicto grupal, la autora plantea tres hipótesis de partida: 1) el avance de la crisis aumenta la competencia por el empleo y el acceso a prestaciones sociales, incrementando la xenofobia; 2) la amenaza económico-material supera a la cultural; y 3) la percepción de la amenaza es más expresa en quiénes se sienten más vulnerables. El análisis longitudinal de los datos empleados por la autora, las encuestas de actitudes hacia la inmigración realizadas entre 2007 y 2012 por el CIS para el Observatorio Español del Racismo y la Xenofobia, matiza las hipótesis planteadas. Así, el artículo muestra que, si bien el avance de la crisis económica ha aumentado la competencia por el empleo y los recursos sociales, este efecto se aprecia especialmente durante los primeros años de recesión. El efecto de la amenaza económico-laboral, al que se apunta desde la teoría del conflicto intergrupal, sí se ha traducido en un aumento de la xenofobia (expresa y tenue) en los momentos de mayor incertidumbre económica y laboral. Al retroceso del rechazo a la inmigración - y la ampliación de la ambivalencia configurada como leve tolerancia- en 2012 puede haber contribuido, en opinión de la autora y como ya apuntaban algunos de sus estudios previos (Cea D'Ancona, 2004), el descenso de la presencia de inmigrantes real y de la presencia percibida por la población. En el cambio experimentado en esta percepción ha influido el reflejo y la imagen que los medios de comunicación ofrecen sobre la inmigración, así como la forma en que esta es tratada en los discursos políticos.

Los datos también confirman que la crisis económica ha contribuido a que la amenaza económico-material supere a la cultural. En este sentido, el 2010 puede ser considerado un año excepcional por el amplio protagonismo político-mediático que en esta fecha tuvo la prohibición del velo islámico en los espacios públicos. Igualmente se confirma que la percepción de amenaza es más expresa en quiénes se sienten más vulnerables. Disfrutar de una buena posición laboral y económica coadyuva a la tolerancia.

Ahondando en esta misma línea de investigación, Sebastian Rinken, en su artículo para este monográfico, emplea una multiplicidad de datos procedentes de diversas encuestas nacionales y autonómicas (entre otros, los datos del CIS y de los Observatorios Andaluz y Vasco de las Migraciones). El artículo de Rinken se detiene en explicar cuáles pueden ser las causas que explican la excepcionalidad 
española como caso que contradice, en cierta medida, la teoría de competencia grupal y su nutrida evidencia empírica. En el período de 2008-2013, la preocupación por el hecho inmigratorio y la animosidad hacia las personas inmigrantes evolucionaron a la baja, pese a un contexto económico que, a priori, hacía presagiar un aumento de la aversión. Se produjo un marcado deterioro de las valoraciones respecto a la dimensión económica de la inmigración, pero se mantuvo un amplio respaldo respecto a la participación política y social de los inmigrantes. Partiendo de esta constatación, el autor identifica cuatro posibles hipótesis de este sorprendente, en su opinión, sosiego ciudadano. Estas explicaciones hacen hincapié en la cultura política de la España post-franquista con su empeño por universalizar los derechos cívicos y sociales; en la inhibición que dicha cultura política supone respecto a la manifestación de prejuicios degradantes; en la desconfianza hacia las élites políticas y su gestión de la crisis, deflactándose así el posible aumento de la animosidad hacia poblaciones de origen inmigrante; y en la percepción de que la crisis afectaría a los inmigrantes con tal crudeza, que muchos de ellos se estarían viendo obligados a re-emigrar.

Más allá de las actitudes ante la inmigración, el análisis sobre la inserción de los inmigrantes en la sociedad española, en un contexto de crisis, pasa necesariamente por analizar el impacto de la recesión en el ámbito escolar, siendo esta una línea escasamente abordada por la producción científica a excepción de algunos trabajos (Marín, Feixa y Nin-Blanco, 2011). El artículo de Javier García Castaño, Antonia Olmos y Ouafaa Bouachra Outmani, para este monográfico, ahonda en esta temática de investigación. Utilizando los datos de la estadística de educación, los autores muestran que durante los últimos cursos se ha producido una moderada disminución de la presencia de población de nacionalidad extranjera en los niveles obligatorios del sistema educativo español. Esta disminución es más acusada en el caso de los nacionales de países latinoamericanos. En la segunda parte del artículo y empleando los datos regionales de Andalucía y de Madrid, los autores constatan la importante reducción observada desde el curso 2009/2010 en algunos de los recursos educativos específicos, en concreto las "Aulas Temporales de Adaptación Lingüística" (ATAL) en Andalucía y las "Aulas de Enlace" en Madrid. Su artículo concluye que la reducción de los recursos escolares específicos para la acogida de menores inmigrantes en el 
sistema educativo de ambas regiones no parece justificarse por la disminución de su volumen.

Queremos terminar señalando que si bien este monográfico ha sacado a la luz las principales temáticas en torno al impacto de la crisis y la inmigración en España, hay otras líneas de investigación igualmente relevantes, que están despertando el interés de los especialistas y que no han sido abordadas por limitaciones de espacio. Entre otras, por señalar algunas de las más importantes, los vínculos entre crisis y política migratoria (Cachón, 2011; López-Sala, 2013), el impacto demográfico del cambio de ciclo migratorio y el resurgimiento de la emigración española (Domingo, Sabater y Ortega, 2014) o el papel de los medios de comunicación y del discurso mediático (Tortajada, Comas y Martínez, 2014). Es necesario también subrayar que la crisis económica ha modulado el debate en torno a la consideración de los migrantes como una carga para los servicios públicos. De ahí el ímpetu de las investigaciones que se han detenido en el entramado existente entre las políticas del bienestar, los recortes sociales y sus efectos sobre la integración y las condiciones de vida de los migrantes (Bruquetas, Marí-Klose y Moreno Fuentes, 2011; Moreno Fuentes y Figueiredo, 2013)

\section{CONCLUSIONES: ¿HACIA LA CONSOLIDACIÓN DE UNA NUEVA AGENDA DE INVESTIGACIÓN?}

El nuevo ciclo migratorio en España ha moldeado una nueva agenda de investigación en el campo de los estudios migratorios. Esto ha supuesto la reformulación de las temáticas, la ampliación de los enfoques, así como la diversificación de las herramientas de aproximación a esta realidad social. De la preponderancia de la medición de los flujos de entrada y de los stocks, de la caracterización de las comunidades y del interés por los retos que han supuesto el establecimiento y la permanencia, los investigadores hemos pasado a centrarnos en los flujos de salida y, con ello, en la emigración, en los procesos de retorno y re-emigración, así como en la diversificación de las formas de movilidad pendular y circular. En resumen, en la complejidad, heterogeneidad y simultaneidad de distintas formas de movilidad que tienen a España como origen o destino y que muestran, una vez más, la necesidad de realizar investigaciones 
multisituadas y poliédricas en donde cada una de las disciplinas pueda realizar aportaciones complementarias.

La incidencia de la crisis en el mercado de trabajo se ha constituido en una línea de investigación clave de la producción científica española, que ha sacado a la luz el impacto diferencial entre autóctonos e inmigrantes, según las diferentes fases de la recesión. $\mathrm{Y}$ que ha puesto igualmente de relieve los diferentes efectos para los hombres y las mujeres, en el marco de un mercado de trabajo segmentado, por género y origen étnico, y abocado a la precariedad. No obstante, más allá de la vulnerabilidad de los inmigrantes ante la crisis económica, los estudiosos de esta realidad social han sacado a luz igualmente la capacidad de agencia y de resiliencia de las comunidades migrantes para hacer frente a la crisis, no sólo a través de decisiones individuales, sino de estrategias familiares. Estas estrategias articulan el ámbito de lo productivo y de lo reproductivo y recubren el campo transnacional. Incorporan nuevas dinámicas laborales y económicas (como el ahorro y reajuste de la gestión de las remesas o el desarrollo de empresas en el nicho étnico), así como nuevas prácticas de movilidad geográfica, tanto interna como internacional. No obstante, más allá de la incidencia del retorno (que no ha llegado a ser tan voluminoso como la crudeza de la crisis habría parecido prever), se constatan formas alternativas de movilidad que se alejan del ortodoxo enfoque bilateral origen-destino y de la hegemonía de la gestión, desde la previsión del carácter permanente de las migraciones. Aspectos que han prevalecido en los análisis migratorios realizados en España durante las dos últimas décadas. El contexto de recesión económica también ha servido para profundizar en los dilemas y los nuevos retos de la convivencia y del uso compartido de espacios y recursos sociales, más aún en un contexto, de acentuados recortes, como los implantados en los últimos años y que han afectado al sector sanitario y educativo. Los estudios especializados han mostrado, sin embargo, la contención en el caso español de la emergencia, como consecuencia de la crisis, de una opinión mayoritariamente contraria a la inmigración vinculada a la competencia por recursos escasos, como la observada en otros países europeos. Esta constatación muestra, una vez más, la excepcionalidad española a la que se han referido otros autores (Arango, 2013), así como su capacidad integradora.

En el campo de la investigación, el actual escenario de incertidumbre y de agudo cambio social y político supone un sustrato de 
un enorme potencial para la maduración y consolidación de los estudios en España, que han ampliado su enfoque desde las lógicas de la recepción y la bonanza a las dinámicas de la emisión y la recesión. Permanecen sin embargo algunas incógnitas que serán, a todas luces, objeto de indagación. Por mencionar algunas de las más relevantes en términos sociales, las respuestas políticas y económicas necesarias para la gestión de la integración en una etapa prolongada de crisis y de contracción del mercado laboral y las futuras dinámicas de la emergente emigración española.

\section{BIBLIOGRAFÍA}

AJA, E; Arango, J. y Oliver, J. (eds.) (2009): La inmigración en tiempos de crisis. Barcelona, CIDOB.

AJA, E; Arango, J. y Oliver, J. (eds.) (2010): Inmigración y crisis económica. Impactos actuales y perspectivas de futuro. Barcelona, CIDOB.

AJA, E; Arango, J. y Oliver, J. (eds.) (2012): Inmigración y crisis. Entre la continuidad y el cambio. Barcelona, CIDOB.

Alemán, C. y Soriano, R. (2013): "Servicios sociales e inmigración en tiempos de crisis económica”. En E. Aja; J. Arango y J. Oliver (eds.), Inmigración y crisis: entre la continuidad y el cambio. Barcelona, CIDOB, pp. 90-115

Allport, F. (1954): The Nature of Prejudice. Cambridge, Adisson-Wesley.

Arango, J. (2013): Exceptional in Europe? Spain's Experience with Immigration and Integration. Washington, Migration Policy Institute.

Aysa-LaStRa, M. y CACHÓn, L. (eds.) (2015): Immigrant Vulnerability and Resilience. Comparative Perspectives on Latin American Immigrants during the Great Recession. Basilea, Springer.

Bastia, T. (2011): "Should I Stay or Should I Go? Return Migration in Times of Crises". Journal of International Development, 23, pp. 583-595.

Blanco, C. (2014): Movilidad humana y diversidad social en un contexto de crisis económica internacional. Madrid, Trotta.

Boccagni, P. y Lagomasino, F. (2011): "Migration and the Global Crisis: New prospects for Return? The case of Ecuadorians in Europe. Bulletin of Latin American Research, 30(3), pp. 282-297. 
Bruquetas, M; Marí-Klose, P. y Moreno Fuentes, J. (2011): “Inmigración, crisis económica y Estado del bienestar en España”. Documentación Social, 162, pp. 209-234.

CACHÓN, L. (2011): “Algunas políticas sobre la inmigración para la crisis y después de la crisis”. En E. Aja, J. Arango y J. Oliver (eds.), Inmigración y crisis económica: impactos actuales y perspectivas de futuro. Barcelona, CIDOB, pp. 252-260.

Cavalcanti, L. y Parella, S. (2013): "El retorno desde una perspectiva transnacional”. REMHU - Revista Interdisciplinar da Mobilidade Humana XXI, 41, pp. 9-20.

CeA D’Ancona, M.A. (2004): La activación de la xenofobia en España. Madrid: CIS/Siglo XXI.

Cebolla, H; Miyar, M. y Muñoz Comet, J. (2015): “Is the Spanish Recession Increasing Inequality? Male migrant-native Differences in Educational Returns against Unemployment”. Journal of Ethnic and Migration Studies, 41(5), pp. 710-728.

Cerase, F. (1974): "Expectations and reality: a case study of return migration from the United States to Southern Italy". International Migration Review, 8(2), pp.245-262.

DE ARce, R. (ed.) (2010): “Retos económicos derivados de la Inmigración en España”. Información Comercial Española, ICE: Revista de economía, 854, pp. 3-4.

Domingo, A. y Sabater, A. (2013): “Crisis económica y emigración”. En E. Aja, J. Arango y J. Oliver (eds.), Inmigración y crisis: entre la continuidad y el cambio. Barcelona, CIDOB, pp. 60-89.

Domingo, A; Sabater, A. y Ortega, E. (2014): “¿ Migración neohispánica? El impacto de la crisis económica en la emigración española». Empiria, 29(1), pp. 39-66.

Domingo A. y Recaño, J. (2010): "La inflexión en el ciclo migratorio internacional en España: impacto y consecuencias demográficas. En E. Aja, J. Arango y J. Oliver (eds.), La inmigración en tiempos de crisis. Barcelona, CIDOB, pp, 182-207.

Dominguez-Mújica, J; Guerra, R. y Parreño, J. (2014): “ Migration at a Time of Global Economic Crisis: The Situation in Spain”. International Migration, 52(6), pp. 113-127.

Durán Ruiz, J; Fernández Avilés, J; Guillén, E; Moreno, N. y Ortega, N. (2011): Inmigración y crisis económica: retos políticos y de ordenación jurídica. Granada, Comares.

Ferrer Sais, A; Ruesga, S. y Resa, C. (2010): Anuario de Relaciones Laborales en España. Volumen 1. Madrid, Unión General de Trabajadores. 
Ferrer Sais, A; Ruesga, S. y Resa, C. (2011): Anuario de Relaciones Laborales en España. Volumen 2. Madrid, Unión General de Trabajadores.

FIELDING, T. (2010): Migration in a Time of Crisis: A simple conceptual framework applied to East Asian migrations. Sussex, University of Sussex. Sussex Centre for Migration Research. Working Paper 63.

Gualda, E. (2012): “Migración circular en tiempos de crisis. Mujeres del Europa del Este y africanas en la agricultura de Huelva”. Papers, 97(3), pp. 613-640.

Garrido, J; Miyar, M. y Muñoz Comet, J. (2010): “La dinámica laboral de los inmigrantes en el cambio de fase del ciclo económico". Presupuesto y Gasto Público, 61, pp. 201-221.

Herrera G. (2012): "Starting Over Again? Crisis, Gender, and Social Reproduction among Ecuadorian Migrants in Spain". Feminist Economics, $18: 2,125-148$

Juliano, D. (2012): "Género y trayectorias migratorias en época de crisis". Papers, 97 (3), pp. 523-540.

Lázaro Alquézar, A; Sánchez, A. y Simón, B. (eds.) (2012): Efectos de la inmigración sobre el empleo en España durante la crisis económica. Santiago de Compostela, XIX Encuentro de Economía Política.

López-SAla, A. (2013): "Managing Uncertainty: Immigration Policies in Spain during Economic Recession (2008-2011)”. Migraciones Internacionales, 7 (2), pp. 39-69.

López-Sala, A. y SÁnchez-Montijano, E. (2014): “Contratación en origen de latinoamericanos en España: un marco flexible de gestión”. Revista d'Afers Internacionals, 106-107, pp. 193-213.

Marin, J; FeiXa, C. y Nin-Blanco, R. (2013): Jóvenes inmigrados en LleidaCataluña, España: transiciones escolares y laborales en un contexto de crisis. Revista Latinoamericana de Ciencias Sociales, 11(2), pp. 493-514.

Matía Portilla, J. y Álvarez Rodríguez, I. (coord.) (2012): Crisis e inmigración. Reflexiones interdisciplinares sobre la inmigración en España. Valencia, Tirant lo Blanch.

Miguélez Lobo, F; López Roldán, P. y Alós-Moner Vila, R. (coord.) (2014): Crisis, empleo e inmigración en España: un análisis de las trayectorias laborales. Barcelona, Universitat Autònoma de Barcelona.

Moreno, G. (2013): “La evolución de las percepciones hacia la inmigración en el País Vasco en el momento de crisis económica: continuidades y rupturas". Revista vasca de Sociología y Ciencia Política, 53-54, pp. 411424. 
Moreno, G. y Fullaondo, A. (2011): “Actitudes y opiniones sobre el uso de prestaciones económicas por parte del colectivo inmigrante en el País Vasco”. Revista de Servicios Sociales, 50, pp. 101-110.

Moreno Fuentes, J. y Figueiredo, S. (2013): “Inmigración, sanidad, crisis económica y politización de la inmigración en España”. Revista Interdisciplinar da Mobilidade Humana, 40, pp. 27-47.

Moser, C. y Horn, P. (2015): "Does economic crisis always harm international migrants? Longitudinal evidence from Ecuadorians in Barcelona”. International Migration, 53(2), pp. 274-290.

Muñoz Comet, J. (2012): “Evolución del empleo y del paro de las mujeres inmigrantes en el mercado de trabajo español. El impacto de la actual crisis económica”. Cuadernos de Relaciones Laborales, 30 (1), pp. 115-137.

Muñoz Comet, J. (2013): "La salida del desempleo de extranjeros y españoles. Efectos del contexto económico”. Revista Española de Investigaciones Sociológicas, 142, pp. 47-70.

Oliver i Alonso, J. (2011): "El mercado de trabajo de la inmigración 2007 - 2010: los cambios 2009/2010 en el marco de la crisis”. En E. Aja, J. Arango y J. Oliver (eds.), Inmigración y crisis económica: impactos actuales y perspectivas de futuro. Barcelona, CIDOB, pp. 130-165.

Oliver i Alonso, J. (2012): "El empleo inmigrante ante la nueva fase de la crisis y la integración de la inmigración”. En E. Aja, J. Arango y J. Oliver (eds.), La hora de la integración. Barcelona, CIDOB, pp. 26-67.

Oliver i Alonso, J. (2013): “La inmigración y la doble recesión del mercado de trabajo en España, 2011-12”. En E. Aja, J. Arango y J. Oliver (eds.), Inmigración y crisis: entre la continuidad y el cambio. Barcelona, CIDOB, pp 28-59.

Oso, L. y CATARINo, C. (2013): "From sex to gender: The feminisation of migration and labour-market insertion in Spain and Portugal". Journal of Ethnic and Migration Studies, 39(4), pp. 625-647.

Oso, L. y Martínez-Buján, R. (2014): "How Latin American immigrant families "protect us" and "one another" in Spain. Strategies of formal and informal social protection within a context of economic crisis". Paper presented at the 11th IMISCOE Annual Conference Immigration, Social Cohesion and Social Innovation, 27-28-29th August 2014, Madrid.

Parella, S. y Petrof, A. (2014): “ Migración de retorno en España: salidas de inmigrantes y programas de retorno en un contexto de crisis”. En J. Arango; D. Moya y J. Oliver (eds.), Inmigración y Emigración: mitos y realidades. Barcelona, CIDOB, pp. 61-87.

Pedone, C.; Agrela, B. y Gil Araujo, S. (2012): “Políticas públicas, migración y familia. Una mirada desde el género”. Papers, 97 (3), pp. 541-568. 
Pedreño, A; Moraes, N. y Gadea, E. (2015): “Crisis, inmigración y desposesión de los servicios públicos”. En F. Torres y E. Gadea (coord.), Crisis, inmigración y sociedad. Madrid, Talasa, pp. 99-128.

Quillian, L. (1995): "Prejudice as response to perceived group threat". American Sociological Review,60(4), pp. 586-612.

Reher, D; Requena, M. y SAnz, A. (2011): “¿España en la encrucijada?: Consideraciones sobre el cambio de ciclo migratorio». Revista Internacional de Sociologia, 69 (1), pp. 9-44.

Rodríguez-Planas, N. y Nollenberger, N. (2014): “Labor Market Integration of New Immigrants in Spain”. Bonn, IZA Policy Papers, 93.

Sánchez Montijano, E. y Alonso, X. (eds.) (2014): Nuevos flujos y la gran recesión: la emigración en Cataluña, España y la Unión Europea. Barcelona, CIDOB.

Sassone, S. y YéPez, I. (comp.) (2014): Monográfico Migración y crisis global. Europa-América Latina: nuevas estrategias, nuevas desigualdades. $R e$ vista d'Afers Internacionals, 106-107.

Torres, F. y Gadea, E.(2015): Crisis, inmigración y sociedad. Madrid, Talasa.

Torres, F. (2014): “Crisis y estrategias de los inmigrantes en España: el acento latino”. Revista d'Afers internacionals, 106-107, pp. 215-236.

Tortajada, I; Comas, D. y Martínez, R. (2014): “Inmigración, crisis económica y discursos radiofónicos: hacia un lenguaje excluyente”. Estudios sobre el Mensaje Periodístico, 20 (2), pp. 899-916.

Viruela, R. (2013): “Entre dos crisis económicas. Estrategias de los rumanos en el mercado de trabajo español”. Política y Sociedad, 50 (3), pp. 9811008. 